\title{
Uncertainty Measurement for the Interval Type-2 Fuzzy Set
}

\author{
Sarah Greenfield ${ }^{1}$ \\ De Montfort University, Leicester, LE1 9BH, UK, \\ s.greenfield@dmu.ac.uk, \\ WWW home page: http://www.tech.dmu.ac.uk/ sarahg/
}

\begin{abstract}
In this paper, two measures of uncertainty for interval type-2 fuzzy sets are presented, evaluated, compared and contrasted. Wu and Mendel regard the length of the type-reduced set as a measure of the uncertainty in an interval set. Greenfield and John argue that the volume under the surface of the type-2 fuzzy set is a measure of the uncertainty relating to the set. For an interval type- 2 fuzzy set, the volume measure is equivalent to the area of the footprint of uncertainty of the set. Experiments show that though the two measures give different results, there is considerable commonality between them. The concept of invariance under vertical translation is introduced; the uncertainty measure of a fuzzy set has the property of invariance under vertical translation if the value it generates remains constant under any vertical translation of the fuzzy set. It is left unresolved whether invariance under vertical translation is an essential property of a type- 2 uncertainty measure.
\end{abstract}

Keywords: interval type-2 fuzzy set, uncertainty, uncertainty bounds, volume measure of uncertainty, invariance under vertical translation

\section{Introduction}

In 1965 Zadeh introduced the concept of the (type-1) fuzzy set 26]. Type-1 membership functions are of questionable accuracy as their derivation tends to be subjective or reliant on large sets of data. The practical application of fuzzy sets is within a Fuzzy Inferencing System (FIS). Uncertainty in type-1 FISs derives from various sources, e.g. "The meanings of the words that are used in the antecedents and consequents of rules can be uncertain" and "Measurements that activate a type-1 FLS may be noisy and therefore uncertain." [19, page 117]. It is therefore very difficult, if not impossible, to determine a type-1 membership function, and consequently it seems somewhat inappropriate to use crisp numbers, possibly expressed to several decimal places, to represent degrees of membership. Klir and Folger [16, page 12] comment:

"... it may seem problematical, if not paradoxical, that a representation of fuzziness is made using membership grades that are themselves precise real numbers. Although this does not pose a serious problem for many 
applications, it is nevertheless possible to extend the concept of the fuzzy set to allow the distinction between grades of membership to become blurred. Sets described in this way are known as type 2 fuzzy sets."

One might argue that the type-1 membership function does reflect the certainty of a proposition. Does not a membership grade of 1 imply certain truth, a grade of 0 certain falsehood, and a grade of 0.5 total uncertainty? But really what is being quantified here is not so much uncertainty as vagueness. This is what lies behind the common use of fuzziness as a so-called measure of uncertainty for type-1 fuzzy sets [23, page 5384].

Zadeh's 1975 innovation of the type-2 fuzzy set 27-29] provides an intuitive model of uncertainty. A type-2 fuzzy set (defined in Subsection 2.2) may be thought of as an adaptation of a type-1 fuzzy set [19, page 118]:

"Imagine blurring the type-1 membership function ... Then, at a specific value of $x$, say $x^{\prime}$, there no longer is a single value for the membership function $\left(u^{\prime}\right)$; instead the membership function takes on values wherever the vertical line intersects the blur. Those values need not all be weighted the same; hence, we can assign an amplitude distribution to all of those points. Doing this for all $x \in X$, we create a three-dimensional membership function - a type-2 membership function - that characterizes a type-2 fuzzy set."

Type-2 fuzzy sets take two forms, generalised, with variable secondary membership grades (Subsection 2.2) between 0 and 1, and the simpler interval, where all secondary membership grades are 1 . The specific concern of this paper is the interval type- 2 fuzzy set. These are increasingly used in applications [3, 5, 6, 11, 13, 15, 17, 20, 21, since interval type-2 fuzzy inferencing is less computationally complex than its generalised counterpart [12, 19].

The concept of a fuzzy uncertainty measure is analogous to that of error bars in statistics. Therefore such a measure has the potential to provide valuable information. By quantifying the uncertainty associated with the aggregated fuzzy set [7, page 1015], one is in effect measuring the uncertainty of the inference generating the aggregated set. In fuzzy image processing [5, 6, 13, for example, an uncertainty measure would indicate the reliability of the processed outputs.

In 23 five measures of uncertainty for interval type-2 fuzzy sets are surveyed, most notably the centroid length measure. A measure not considered in this survey is that of the area of the interval set's Footprint Of Uncertainty (FOU). In this report the centroid length and the FOU area measures are compared and contrasted.

The next section covers preliminaries such as assumptions and definitions. Following that, in Section 3, the two uncertainty measures are presented, after which, in Section 4, the experiments by which the methods are compared and contrasted are described and their implications assessed. Finally Section 5 concludes the paper. 


\section{Preliminaries}

\subsection{Assumptions}

The following assumptions relate to fuzzy sets:

1. The type-1 fuzzy set is contained within a unit square and may be viewed as a curve represented by $(x, u)$ co-ordinates.

2. The type-2 fuzzy set is contained within a unit cube and may be viewed as a surface represented by $(x, u, z)$ co-ordinates.

3. The domain ( $x$-axis) is discretised.

\subsection{Definitions}

Let $X$ be a universe of discourse. A type- 1 fuzzy set $A$ on $X$ is characterised by a membership function $\mu_{A}: X \rightarrow[0,1]$ and can be expressed as follows [26]:

$$
A=\left\{\left(x, \mu_{A}(x)\right) \mid \mu_{A}(x) \in[0,1] \forall x \in X\right\} .
$$

In the following the notation $U=[0,1]$ is employed.

Let $\tilde{P}(U)$ be the set of fuzzy sets in $U$. A type- 2 fuzzy set $\tilde{A}$ in $X$ is a fuzzy set whose membership grades are themselves fuzzy [27-29]. This implies that $\mu_{\tilde{A}}(x)$ is a fuzzy set in $U$ for all $x$, i.e. $\mu_{\tilde{A}}: X \rightarrow \tilde{P}(U)$ and

$$
\tilde{A}=\left\{\left(x, \mu_{\tilde{A}}(x)\right) \mid \mu_{\tilde{A}}(x) \in \tilde{P}(U) \forall x \in X\right\} .
$$

It follows that $\forall x \in X \exists J_{x} \subseteq U$ such that $\mu_{\tilde{A}}(x): J_{x} \rightarrow U$. Applying (1), we obtain:

$$
\mu_{\tilde{A}}(x)=\left\{\left(u, \mu_{\tilde{A}}(x)(u)\right) \mid \mu_{\tilde{A}}(x)(u) \in U \forall u \in J_{x} \subseteq U\right\} .
$$

$X$ is called the primary domain and $J_{x}$ the primary membership of $x$ while $U$ is known as the secondary domain and $\mu_{\tilde{A}}(x)$ the secondary membership of $x$.

Putting (2) and (3) together, we obtain the definition of a generalised type-2 fuzzy set:

$$
\tilde{A}=\left\{\left(x,\left(u, \mu_{\tilde{A}}(x)(u)\right)\right) \mid \mu_{\tilde{A}}(x)(u) \in U, \forall x \in X \wedge \forall u \in J_{x} \subseteq U\right\} .
$$

Definition 1 (Interval Type-2 Fuzzy Set). An interval type-2 fuzzy set is a type-2 fuzzy set whose secondary membership grades are all 1.

In the interval case, Equation 4 reduces to:

$$
\tilde{A}=\left\{(x,(u, 1)), \quad \forall x \in X \wedge \forall u \in J_{x} \subseteq U\right\} .
$$

Definition 2 (Footprint Of Uncertainty [19]). The Footprint Of Uncertainty (FOU) is the projection of the type-2 fuzzy set onto the $x-u$ plane.

The FOU defines the interval set, as all its secondary membership grades are 1 . 
Definition 3 (Lower Membership Function). The Lower Membership Function (LMF) of a type-2 fuzzy set is the type-1 membership function associated with the lower bound of the FOU.

Definition 4 (Upper Membership Function). The Upper Membership Function (UMF) of a type-2 fuzzy set is the type-1 membership function associated with the upper bound of the FOU.

Discretisation is the process by which a continuous set is converted into a discrete set through a process of slicing.

Definition 5 (Vertical Slice [19]). A vertical slice of a type-2 fuzzy set is a plane through the $x$-axis, parallel to the $u-z$ plane.

Definition 6 (Degree of Discretisation). The degree of discretisation is the separation of the slices.

Definition 7 (Rectangular Type-2 Fuzzy Set). A rectangular type-2 fuzzy set is an interval type-2 fuzzy set whose FOU extends between the lines $x=0$ and $x=1$, with $L M F$ and $U M F$ both running parallel to the $x$-axis.

Figure 1 depicts two rectangular type-2 fuzzy sets.

Definition 8 (Blank Type-2 Fuzzy Set). The blank type-2 fuzzy set is a rectangular type-2 fuzzy set whose LMF is the line $u=0$ and UMF is the line $u=1$.

Definition 9 (Invariance under Vertical Translation). An attribute of a fuzzy set has the property of Invariance under Vertical Translation (IVT) if it remains constant under any vertical translation of the fuzzy set.

Definition 10 (Embedded Set). An embedded set is a special kind of type-2 fuzzy set, which relates to the type-2 fuzzy set in which it is embedded in this way: For every primary domain value, $x$, there is a unique secondary domain value, $u$, plus the associated secondary membership grade that is determined by the primary and secondary domain values, $\mu_{\tilde{A}}(x)(u)$.

The centroid length uncertainty measure is inextricably linked with the defuzzification process of a type-2 fuzzy set. Type-reduction, the first stage of type-2 defuzzification, creates a type- 1 fuzzy set know as the Type-Reduced Set (TRS). Assuming that the primary domain $X$ has been discretised, the TRS of a type-2 fuzzy set may be defined thus [27, [19, page 121]:

Definition 11. The TRS associated with a type-2 fuzzy set $\tilde{A}$ with primary domain $X$ discretised into $N$ points $X=\left\{x_{1}, x_{2}, \ldots, x_{N}\right\}$, is

$$
\begin{aligned}
C_{\tilde{A}}= & \left\{\left(\frac{\sum_{i=1}^{N} x_{i} \cdot u_{k_{i}}}{\sum_{i=1}^{N} u_{k_{i}}}, \mu_{\tilde{A}}\left(x_{1}\right)\left(u_{k_{1}}\right) * \ldots * \mu_{\tilde{A}}\left(x_{N}\right)\left(u_{k_{N}}\right)\right) \mid \forall\left(u_{k_{1}}, u_{k_{2}}, \ldots, u_{k_{N}}\right)\right. \\
& \left.\in J_{x_{1}} \times J_{x_{2}} \times \ldots \times J_{x_{N}} \subseteq U^{N}\right\} .
\end{aligned}
$$


For the TRS of an interval type-2 fuzzy set, Definition 11 reduces to:

Definition 12 (TRS of an Interval Type-2 Set). The TRS associated with an interval type-2 fuzzy set $\tilde{A}$ with primary domain $X$ discretised into $N$ points $X=\left\{x_{1}, x_{2}, \ldots, x_{N}\right\}$, is

$C_{\tilde{A}}=\left\{\left(\frac{\sum_{i=1}^{N} x_{i} \cdot u_{k_{i}}}{\sum_{i=1}^{N} u_{k_{i}}}, 1\right) \mid \forall\left(u_{k_{1}}, u_{k_{2}}, \ldots, u_{k_{N}}\right) \in J_{x_{1}} \times J_{x_{2}} \times \ldots \times J_{x_{N}} \subseteq U^{N}\right\}$.

Mendel and John's Representation Theorem [19, page 121] provides a precise method for defuzzification of type-2 fuzzy sets. Though Definitions 11 and 12 do not explicitly mention embedded sets, they appear implicitly in Equations 6 and 7. When these equations are presented in algorithmic form (Algorithm 1), they are explicitly referred to. Exhaustive type-reduction (Algorithm 1) processes every embedded set in turn, hence the term 'exhaustive method' [, 9]. Each embedded set is defuzzified as a type- 1 fuzzy set. The defuzzified value is paired with the minimum secondary membership grade of the embedded set, which in the interval case is 1 , as all the secondary membership grades are 1 . The set of ordered pairs constitutes the TRS, which is then defuzzified as a type- 1 fuzzy set to give the defuzzified value of the type-2 fuzzy set.

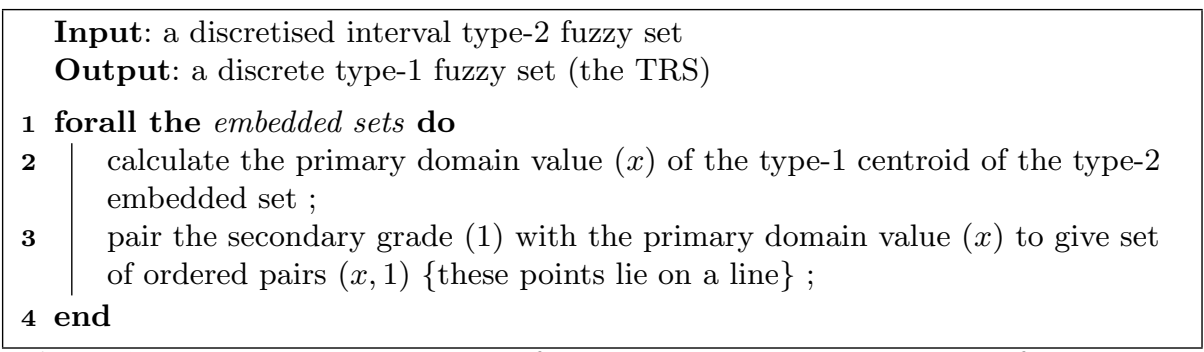

Algorithm 1: Type-reduction of an interval discretised type-2 fuzzy set to a type-1 fuzzy set, adapted from Mendel [18.

\subsection{Principle of Type-2 Uncertainty Measurement}

Mendel [18, page 11] advocates the fundamental design requirement with regard to type-2 uncertainty measurement: "When all sources of uncertainty disappear, a type-2 FLS must reduce to a comparable type-1 FLS." This principle is patently valid.

It follows from this requirement that there is no uncertainty associated with a type-1 fuzzy set, and hence so called measures of uncertainty for type-1 fuzzy sets [1, 2, 4, 22] cannot be measuring uncertainty; perhaps they are measuring another characteristic of the type-1 set such as vagueness (Section 1). 


\section{Type-2 Uncertainty Measures}

In this section the two interval type-2 uncertainty measures of TRS length and FOU area are presented; these measures contrast strongly in their rationales and derivations.

\subsection{Length of the TRS as a Measure of Uncertainty}

$\mathrm{Wu}$ and Mendel propose that the length of the TRS of an interval set provides a measure of the uncertainty of the set 25. The most widely adopted method for type-reducing an interval type-2 fuzzy set is the Karnik-Mendel Iterative Procedure $(K M I P)$ [14. The result of type-reduction of an interval type-2 fuzzy set is an interval (a particular case of a type-1 fuzzy set), with the defuzzified value at the midpoint. The endpoints of the interval are termed uncertainty bounds [25, page 622]. The iterative procedure is an efficient search method for locating these endpoints. It is an approximate technique [7], [14, page 203]. Since the publication of the KMIP, various more efficient versions have been proposed [24, which differ somewhat in their search strategy whilst giving the same result. However, in this paper the absolutely accurate Exhaustive Method [7] is used. Though it has relatively high computational complexity, in the experiments described below the discretisation employed is coarse enough for defuzzification to be accomplished relatively speedily.

\subsection{Area of the FOU as a Measure of Uncertainty}

How type-2 fuzzy sets model uncertainty is the subject of [10]. In this book chapter it is proposed that the third dimension reflects the uncertainty arising out of a deficit in information. From this premise it is argued that the volume under the surface of the type- 2 fuzzy set is a measure of the uncertainty relating to the set. For an interval type- 2 fuzzy set, since the secondary membership grades all take the value of 1 , the area of the FOU is equivalent to the volume under the surface of the type- 2 fuzzy set. The measure is applied to the aggregated fuzzy set [7, page 1015]; this in effect measures the uncertainty of the inference from which the aggregated set is generated (Section 1).

Minimum Uncertainty The least amount of uncertainty possible is 0. This corresponds to a type-2 fuzzy set in which every secondary membership function is a vertical line of height 1 , with 0 area, originating from an FOU that is a line. Such a type-2 fuzzy set is equivalent to, and reducible to, a type-1 fuzzy set.

Maximum Uncertainty At the other extreme, the greatest amount of uncertainty possible is 1 . There is only one type- 2 fuzzy set having uncertainty of 1 , an interval set for which the support for each vertical slice's secondary membership function is the complete interval $[0,1]$. The area of the FOU is 1 . This type- 2 fuzzy set may be described as a unit cube (of volume 1). It is fitting that it has an uncertainty of 1 , as, being essentially formless, like a blank sheet of paper, it is devoid of information. For this reason it is termed the blank type-2 fuzzy set. 


\section{Experiments}

\subsection{Methodology}

The two uncertainty measures are compared and contrasted experimentally, using specially constructed test sets. There are four experimental test runs. For three of the test runs rectangular interval type-2 fuzzy sets (Figure 2) are employed. For the fourth test run, the underlying test set has no specific symmetry or form (Figure 1). The strategy adopted is to either alter the distance between the LMF and the UMF, or keep this distance constant whilst translating the test set vertically, in each instance applying both the centroid length and the FOU area measures. The accurate Exhaustive Method [7] is used to generate the TRS, as opposed to the KMIP [14], which is an approximation. For all the test sets the domain has a degree of discretisation of 0.1 , so engendering 11 evenly spaced vertical slices.
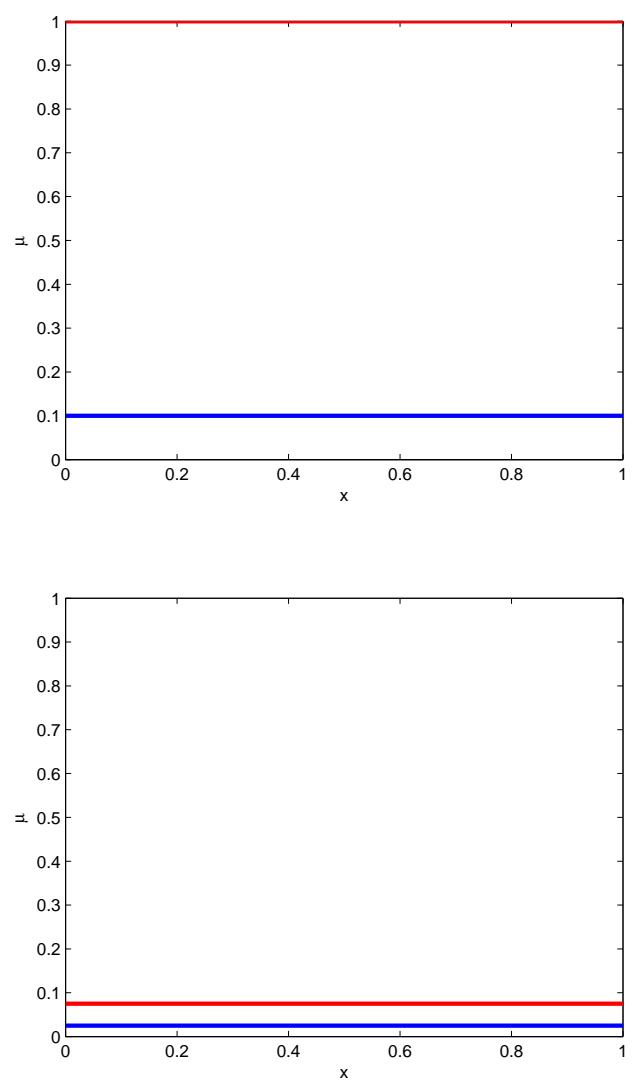

Fig. 1. Rectangular type-2 fuzzy test sets. The LMF and UMF are shown as bold lines. 

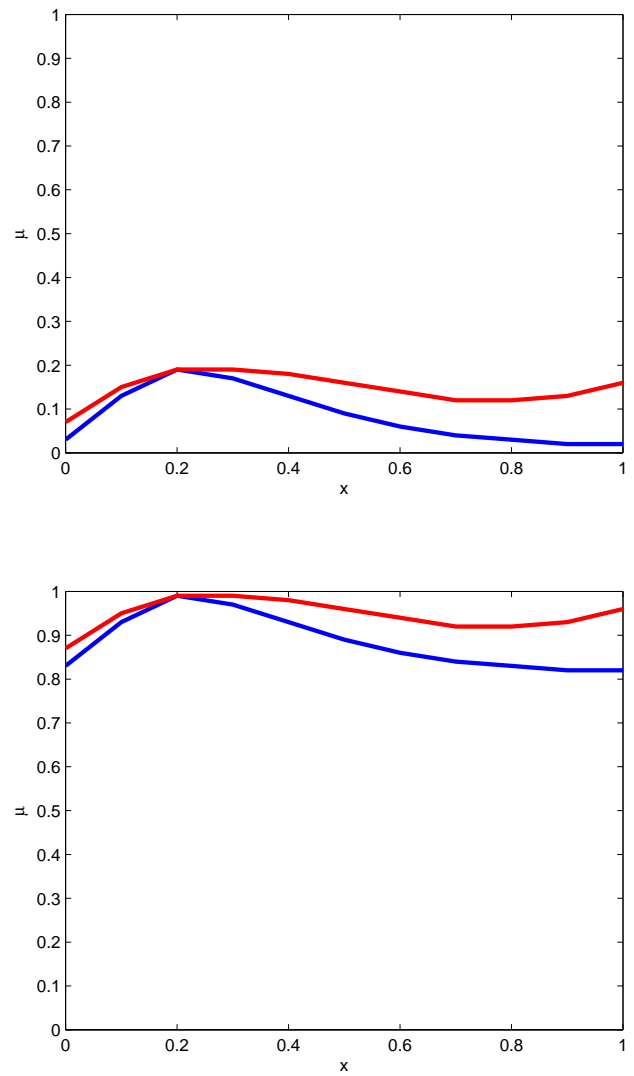

Fig. 2. Asymmetric type-2 fuzzy test sets. The LMF and UMF are shown as bold lines.

\subsection{Results}

The results of the experiments are presented in Tables 1 to 4.

There are several points of commonality between the two measures:

1. From the trend in the last two columns of Table 2, the minimum uncertainty for both measures is evidently 0 .

2. From the trend in the last two columns of Table 1 , the maximum uncertainty for both measures is evidently 1 .

3. If the distance between the LMF and UMF is increased, the amount of uncertainty increases, as measured by both TRS length and FOU area (Tables 1 and 2 .

4. As the distance between the LMF and UMF decreases to 0 (Table 2), the TRS length and FOU area both decrease to 0 . Both measures tend to the value 0 as representing no uncertainty i.e. total certainty, as for a type-1 fuzzy set. 


\begin{tabular}{|l|l|l|l|l|l|}
\hline LMF & UMF & $\begin{array}{l}\text { Left Uncert. } \\
\text { Bound }\end{array}$ & $\begin{array}{l}\text { Right Uncert. } \\
\text { Bound }\end{array}$ & $\begin{array}{l}\text { Length } \\
\text { of TRS }\end{array}$ & $\begin{array}{l}\text { Area } \\
\text { of FOU }\end{array}$ \\
\hline 0.1 & 1 & 0.2157894737 & 0.7842105263 & 0.5684210526 & 0.9000000000 \\
0.01 & 1 & 0.0500000000 & 0.9500000000 & 0.9000000000 & 0.9900000000 \\
0.001 & 1 & 0.0054455446 & 0.9945544554 & 0.9891089109 & 0.9990000000 \\
0.0001 & 1 & 0.0005494505 & 0.9994505495 & 0.9989010989 & 0.9999000000 \\
0.00001 & 1 & 0.0000549945 & 0.9999450055 & 0.9998900110 & 0.9999900000 \\
0.000001 & 1 & 0.0000054999 & 0.9999945001 & 0.9999890001 & 0.9999990000 \\
0.0000001 & 1 & 0.0000005500 & 0.9999994500 & 0.9999989000 & 0.9999999000 \\
0.00000001 & 1 & 0.0000000550 & 0.9999999450 & 0.9999998900 & 0.9999999900 \\
0.000000001 & 1 & 0.0000000055 & 0.9999999945 & 0.9999999890 & 0.9999999990 \\
0.0000000001 & 1 & 0.0000000005 & 0.9999999994 & 0.9999999989 & 0.9999999999 \\
\hline
\end{tabular}

Table 1. Rectangular test sets with defuzzified values of 0.5000000000 . The UMF is constant at 1 , and the LMF decreases from 0.1 towards 0 .

\begin{tabular}{|l|l|l|l|l|l|}
\hline LMF & UMF & $\begin{array}{l}\text { Left Uncert. } \\
\text { Bound }\end{array}$ & $\begin{array}{l}\text { Right Uncert. } \\
\text { Bound }\end{array}$ & $\begin{array}{l}\text { Length } \\
\text { of TRS }\end{array}$ & $\begin{array}{l}\text { Area } \\
\text { of FOU }\end{array}$ \\
\hline 0.9 & 1 & 0.4855769231 & 0.5144230769 & 0.0288461538 & 0.1000000000 \\
0.99 & 1 & 0.4986288848 & 0.5013711152 & 0.0027422303 & 0.0100000000 \\
0.999 & 1 & 0.4998635619 & 0.5001364381 & 0.0002728761 & 0.0010000000 \\
0.9999 & 1 & 0.4999863629 & 0.5000136371 & 0.0000272742 & 0.0001000000 \\
0.99999 & 1 & 0.4999986364 & 0.5000013636 & 0.0000027273 & 0.0000100000 \\
0.999999 & 1 & 0.4999998636 & 0.5000001364 & 0.0000002727 & 0.0000010000 \\
0.9999999 & 1 & 0.4999999864 & 0.5000000136 & 0.0000000273 & 0.0000001000 \\
0.99999999 & 1 & 0.4999999986 & 0.5000000014 & 0.0000000027 & 0.0000000100 \\
0.999999999 & 1 & 0.4999999999 & 0.5000000001 & 0.0000000003 & 0.0000000010 \\
\hline
\end{tabular}

Table 2. Rectangular test sets with defuzzified values of 0.5000000000 . The UMF is constant at 1 , and the LMF increases from 0.9 towards 1.

\begin{tabular}{|l|l|l|l|l|l|}
\hline LMF & UMF & $\begin{array}{l}\text { Left Uncert. } \\
\text { Bound }\end{array}$ & $\begin{array}{l}\text { Right Uncert. } \\
\text { Bound }\end{array}$ & $\begin{array}{l}\text { Length } \\
\text { of TRS }\end{array}$ & $\begin{array}{l}\text { Area } \\
\text { of FOU }\end{array}$ \\
\hline 0.025 & 0.075 & 0.3526315789 & 0.6473684211 & 0.2947368421 & 0.0500000000 \\
0.125 & 0.175 & 0.4538461538 & 0.5461538462 & 0.0923076923 & 0.0500000000 \\
0.225 & 0.275 & 0.4724770642 & 0.5275229358 & 0.0550458716 & 0.0500000000 \\
0.325 & 0.375 & 0.4803921569 & 0.5196078431 & 0.0392156863 & 0.0500000000 \\
0.425 & 0.475 & 0.4847715736 & 0.5152284264 & 0.0304568528 & 0.0500000000 \\
0.525 & 0.575 & 0.4875518672 & 0.5124481328 & 0.0248962656 & 0.0500000000 \\
0.625 & 0.675 & 0.4894736842 & 0.5105263158 & 0.0210526316 & 0.0500000000 \\
0.725 & 0.775 & 0.4908814590 & 0.5091185410 & 0.0182370821 & 0.0500000000 \\
0.825 & 0.875 & 0.4919571046 & 0.5080428954 & 0.0160857909 & 0.0500000000 \\
0.925 & 0.975 & 0.4928057554 & 0.5071942446 & 0.0143884892 & 0.0500000000 \\
\hline
\end{tabular}

Table 3. Rectangular test sets with defuzzified values of 0.5000000000 . The distance between the LMF and UMF is constant at 0.05 , but the height of the test set increases. 


\begin{tabular}{|l|l|l|l|l|l|l|}
\hline $\begin{array}{l}\text { Min. } \\
\text { LMF }\end{array}$ & $\begin{array}{l}\text { Max. } \\
\text { UMF }\end{array}$ & $\begin{array}{l}\text { Left Uncert. } \\
\text { Bound }\end{array}$ & $\begin{array}{l}\text { Right Uncert. } \\
\text { Bound }\end{array}$ & $\begin{array}{l}\text { Defuzzified } \\
\text { Value }\end{array}$ & $\begin{array}{l}\text { Length } \\
\text { of TRS }\end{array}$ & $\begin{array}{l}\text { Area } \\
\text { of FOU }\end{array}$ \\
\hline 0.02 & 0.19 & 0.3363636364 & 0.5248226950 & 0.4442324244 & 0.1884590587 & 0.0610000000 \\
0.22 & 0.39 & 0.4484567901 & 0.5096952909 & 0.4805290824 & 0.0612385007 & 0.0610000000 \\
0.42 & 0.59 & 0.4693014706 & 0.5060240964 & 0.4881952415 & 0.0367226258 & 0.0610000000 \\
0.62 & 0.79 & 0.4781413613 & 0.5043695381 & 0.4915292610 & 0.0262281768 & 0.0610000000 \\
0.82 & 0.99 & 0.4830284553 & 0.5034280118 & 0.4933946116 & 0.0203995565 & 0.0610000000 \\
\hline
\end{tabular}

Table 4. Asymmetric test sets with variable defuzzified values. The shape and size of the test set is constant, but the height of the test set increases.

5. As the distance between the LMF and UMF increases to 1 (Table 1), the TRS length and FOU area both increase to 1 . Both measures tend to the value 1 as indicating maximum uncertainty, as for a blank fuzzy set.

Nonetheless there are clear discrepancies in the values reached by the two measures; they are not equivalent. If an interval set is translated in the $x-u$ plane so that its $u$ co-ordinate is increased, its uncertainty decreases, as measured by TRS length, yet the FOU area measure remains constant. There is thus an extreme contrast between the two measures in regard to IVT (Definition 9), as the FOU area measure adheres absolutely to IVT, whereas the TRS length measure does not. Is IVT an essential characteristic for a type-2 fuzzy uncertainty measure? In other words, should IVT be a design requirement? If so, then TRS length is unacceptable as an uncertainty measure; if not TRS length is a valid measure of uncertainty.

\section{Conclusion}

Two measures for the uncertainty relating to an interval type-2 fuzzy set are examined in this paper. Though as techniques they totally contrast, the experiments presented show that similarities are nonetheless apparent in their behaviour.

The most telling difference in the outcomes of the two measurement techniques is in relation to invariance under vertical translation, a characteristic which the FOU area measure adheres to, but the TRS length measure does not. Whether IVT is an essential characteristic for a type-2 fuzzy uncertainty measure is left unresolved, a suitable topic for further work. 


\section{Bibliography}

[1] Bonissone, P.P.: A pattern recognition approach to the problem of linguistic approximation. In: Proceedings of the IEEE International Conference on Cybernetics and Society. pp. 793 - 798. Denver, Colorado, USA (1979)

[2] Bonissone, P.P.: A fuzzy sets based linguistic approach: theory and applications. In: Proceedings of the 12th Winter Simulation Conference. pp. 99 - 111. Orlando, Florida, USA (1980)

[3] Boumella, N., Djouani, K., Boulemden, M.: On an Interval Type-2 TSK FLS A1-C1 Consequent Parameters Tuning. In: Proceedings of the IEEE Symposium on Advances in Type-2 Fuzzy Logic Systems 2011. Paris (April 2011)

[4] Dubois, D., Prade, H.: Fuzzy cardinality and the modeling of imprecise quantification. Fuzzy Sets and Systems 16, 199 - 230 (1985)

[5] Fisher, P.F.: Remote sensing of land cover classes as type 2 fuzzy sets. Remote Sensing of Environment 114(2), 309

[6] Galar, M., Barrenechea, E., Fernandez, J., Bustince, H., Beliakov, G.: Representing Images by Means of Interval-Valued Fuzzy Sets. Application to Stereo Matching. In: Proceedings of the IEEE Symposium on Advances in Type-2 Fuzzy Logic Systems 2011. Paris (April 2011)

[7] Greenfield, S., Chiclana, F.: Accuracy and Complexity Evaluation of Defuzzification Strategies for The Discretised Interval Type-2 Fuzzy Set. International Journal of Approximate Reasoning 54(8), 1013 - 1033 (October 2013), DOI: http://dx.doi.org/10.1016/j.ijar.2013.04.013

[8] Greenfield, S., Chiclana, F.: Defuzzification of the Discretised Generalised Type-2 Fuzzy Set: Experimental Evaluation. Information Sciences 244, 1 25 (September 2013), DOI: http://dx.doi.org/10.1016/j.ins.2013.04.032

[9] Greenfield, S., Chiclana, F., John, R.I.: Type-Reduction of the Discretised Interval Type-2 Fuzzy Set. In: Proceedings of FUZZ-IEEE 2009. pp. 738743. Jeju Island, Korea (August 2009)

[10] Greenfield, S., John, R.I.: The Uncertainty Associated with a Type-2 Fuzzy Set. In: Rudolf Seising (editor) Views on Fuzzy Sets and Systems from Different Perspectives, in 'Studies in Fuzziness and Soft Computing', series editor Janusz Kacprzyk. vol. 243, pp. 471-483. Springer-Verlag (2009), DOI: http://dx.doi.org/10.1007/978-3-540-93802-6_23

[11] Hagras, H., Wagner, C.: Introduction to Interval Type-2 Fuzzy Logic Controllers - Towards Better Uncertainty Handling in Real World Applications. IEEE Systems, Man and Cybernetics eNewsletter (2009), issue 27

[12] John, R.I., Coupland, S.: Type-2 Fuzzy Logic: A Historical View. IEEE Computational Intelligence Magazine 2(1), 57 - 62 (February 2007), DOI: 10.1109/MCI.2007.357194

[13] Jurio, A., Paternain, D., Lopez-Molina, C., Bustince, H., Mesiar, R., Beliakov, G.: A Construction Method of Interval-Valued Fuzzy Sets for Image 
Processing. In: Proceedings of the IEEE Symposium on Advances in Type-2 Fuzzy Logic Systems 2011. Paris (April 2011)

[14] Karnik, N.N., Mendel, J.M.: Centroid of a Type-2 Fuzzy Set. Information Sciences 132, $195-220$ (2001)

[15] Kayacan, E., Cigdem, O., Kaynak, O.: On Novel Training Method Based on Variable Structure Systems Approach for Interval Type-2 Fuzzy Neural Networks. In: Proceedings of the IEEE Symposium on Advances in Type-2 Fuzzy Logic Systems 2011. Paris (April 2011)

[16] Klir, G.J., Folger, T.A.: Fuzzy Sets, Uncertainty, and Information. PrenticeHall International (1992)

[17] Leottau, L., Melgarejo, M.: Implementing an Interval Type-2 Fuzzy Processor onto a DSC 56F8013. In: Proceedings of FUZZ-IEEE 2010. pp. 19391942. Barcelona, Spain (2010)

[18] Mendel, J.M.: Uncertain Rule-Based Fuzzy Logic Systems: Introduction and New Directions. Prentice-Hall PTR (2001)

[19] Mendel, J.M., John, R.I.: Type-2 fuzzy sets made simple. IEEE Transactions on Fuzzy Systems 10(2), 117 - 127 (2002), DOI: http://dx.doi.org/10.1109/91.995115

[20] Sanz, J.A., Fernández, A., Bustince, H., Herrera, F.: A Genetic Tuning to Improve the Performance of Fuzzy Rule-Based Classification Systems with Interval-Valued Fuzzy Sets: Degree of Ignorance and Lateral Position. International Journal of Approximate Reasoning 52, 751-766 (2011)

[21] Tellez-Velazquez, A., Molina-Lozano, H., Moreno-Armendariz, M.A., Rubio-Espino, E., Villa-Vargas, L.A., Batyrshin, I.: Parametric Type-2 Fuzzy Control Design for the Ball and Plate System. In: Proceedings of the IEEE Symposium on Advances in Type-2 Fuzzy Logic Systems 2011. Paris (April 2011)

[22] Wenstøp, F.: Quantitative analysis with linguistic values. Fuzzy Sets and Systems 4, $99-115$ (1980)

[23] Wu, D., Mendel, J.M.: Uncertainty measures for interval type-2 fuzzy sets. Information Sciences 177, 5378 - 5393 (July 2007)

[24] Wu, D., Mendel, J.M.: Enhanced Karnik-Mendel Algorithms. IEEE Transactions on Fuzzy Systems 17(4), 923 - 934 (August 2009)

[25] Wu, H., Mendel, J.M.: Uncertainty Bounds and Their Use in the Design of Interval Type-2 Fuzzy Logic Systems. IEEE Transactions on Fuzzy Systems 10(5), 622-639 (2002)

[26] Zadeh, L.A.: Fuzzy Sets. Information and Control 8, 338 - 353 (1965)

[27] Zadeh, L.A.: The Concept of a Linguistic Variable and its Application to Approximate Reasoning. Information Sciences 8, 199 - 249 (1975)

[28] Zadeh, L.A.: The Concept of a Linguistic Variable and its Application to Approximate Reasoning - II. Information Sciences 8, 301 - 357 (1975)

[29] Zadeh, L.A.: The Concept of a Linguistic Variable and its Application to Approximate Reasoning - III. Information Sciences 9, 43 - 80 (1975) 